\title{
FIXED ALGEBRAS OF RESIDUALLY NILPOTENT LIE ALGEBRAS
}

\author{
VESSELIN DRENSKY
}

(Communicated by Maurice Auslander)

\begin{abstract}
Let $L_{m}$ be the free Lie algebra of rank $m>1$ over a field $K$, and let $J$ be an ideal of $L_{m}$ such that $J \subset L_{m}^{\prime \prime}$ and the algebra $L_{m} / J$ is residually nilpotent. Let $G \neq\langle 1\rangle$ be a finite group of automorphisms of $L_{m} / J$ and the order of $G$ be invertible in $K$. We establish that the algebra of fixed points $\left(L_{m} / J\right)^{G}$ is not finitely generated. The class of algebras under consideration contains the free Lie algebra over an arbitrary field and the relatively free algebras in nonnilpotent varieties of Lie algebras over infinite fields of characteristic different from 2 and 3.
\end{abstract}

\section{INTRODUCTION}

By a result of Dyer and Scott [4, Theorem 2], for any finite group $G$ of automorphisms of the free group $F_{m}$ of finite rank, the subgroup of fixed points

$$
F_{m}^{G}=\left\{f \in F_{m} \mid g(f)=f \text { for all } g \in G\right\}
$$

is finitely generated. For the free associative algebra $K\left\langle x_{1}, \ldots, x_{m}\right\rangle$ over a field $K$ of characteristic 0 the situation is completely different. Dicks and Formanek [2, Theorem 5.3] and Kharchenko [6, Theorem 2] have established that, for a finite group $G$ acting linearly on $\operatorname{span}\left\{x_{1}, \ldots, x_{m}\right\}$, the algebra of invariants $K\left\langle x_{1}, \ldots, x_{m}\right\rangle^{G}$ is finitely generated if and only if $G$ acts by scalar multiplication on the generating set $\left\{x_{1}, \ldots, x_{m}\right\}$.

The starting point of this research was the problem for finite generation of the fixed algebra of the free Lie algebra $L_{m}$ of rank $m>1$ over an arbitrary field $K$. The only result known is due to Belov [1] who has proved that $L_{m}^{G}$ is not finitely generated if $G \neq\langle 1\rangle$ is a finite cyclic subgroup of Aut $L_{m}$ and the order of $G$ is invertible in $K$. Our main result is the following.

Let $J$ be an ideal of $L_{m}, m>1$, such that $J$ is contained in $L_{m}^{\prime \prime}=$ $\left[\left[L_{m}, L_{m}\right],\left[L_{m}, L_{m}\right]\right]$, and let the algebra $L_{m} / J$ be residually nilpotent, i.e.,

$$
\bigcap_{n \geq 1}\left(L_{m} / J\right)^{n}=\{0\}, \quad \text { where }\left(L_{m} / J\right)^{n}=\underbrace{\left[L_{m} / J, \ldots, L_{m} / J\right]}_{n} .
$$

Received by the editors February 3, 1992 and, in revised form, July 16, 1992.

1991 Mathematics Subject Classification. Primary 17B40; Secondary 15A72, 17B01, 17B30.

Key words and phrases. Fixed points of automorphisms of Lie algebras, residually nilpotent Lie algebras, free Lie algebras.

This research was carried out when the author was an Alexander von Humboldt fellow in the University of Bielefeld, Germany. 
For any finite subgroup $G \neq\langle 1\rangle$ of the automorphism group $\operatorname{Aut}\left(L_{m} / J\right)$ such that the order of $G$ is invertible in $K$, the fixed algebra

$$
\left(L_{m} / J\right)^{G}=\left\{f \in L_{m} / J \mid g(f)=f \text { for all } g \in G\right\}
$$

is not finitely generated.

The following algebras belong to the class under consideration:

(i) The free Lie algebra $L_{m}$.

(ii) The relatively free algebras $F_{m}(\mathfrak{U})$ in nonnilpotent varieties $\mathfrak{U}$ of Lie algebras over infinite fields of characteristic different from 2 and 3.

Our approach is based on noncommutative invariant theory of finite linear groups and essentially depends on the Maschke theorem. First we solve the problem for the free metabelian Lie algebra and then, as a consequence, for any algebra from the class under consideration. To the best of our knowledge, the problem for finite generation of $L_{m}^{G}$ when char $K$ divides $|G|$ is still open.

\section{Preliminaries}

Let $K$ be an arbitrary field, and let $L_{m}$ be the free Lie algebra of rank $m>1$ freely generated by $x_{1}, \ldots, x_{m}$. We use left normed notation for the commutators in $L_{m}$ :

$$
\left[u_{1}, u_{2}\right]=u_{1}\left(\operatorname{ad} u_{2}\right), \quad\left[u_{1}, \ldots, u_{n-1}, u_{n}\right]=\left[\left[u_{1}, \ldots, u_{n-1}\right], u_{n}\right] .
$$

Let $V_{m}=\operatorname{span}\left\{x_{1}, \ldots, x_{m}\right\}$ be the vector space spanned on the free generators of $L_{m}$. The natural action of the general linear group $\mathrm{GL}_{m}=\mathrm{GL}\left(V_{m}\right)$ on $V_{m}$ can be extended diagonally on $L_{m}$. Let $U$ be an ideal of $L_{m}$ which is closed under all endomorphisms of $L_{m} ; U$ is called a verbal ideal. The class $\mathfrak{U}$ of all Lie algebras satisfying the polynomial identities from $U$ is called a variety of algebras, and the factor-algebra $F_{m}(\mathfrak{U})=L_{m} / U$ is the relatively free algebra of rank $m$ in $\mathfrak{U}$. We use the same symbols $x_{1}, \ldots, x_{m}$ for the generators of $F_{m}(\mathfrak{U})$. Clearly $F_{m}(\mathfrak{U})$ inherits the $\mathrm{GL}_{m}$-module structure of $L_{m}$.

Let $\operatorname{char} K=0$. For a partition $\lambda=\left(\lambda_{1}, \ldots, \lambda_{m}\right)$ (i.e., $\lambda_{1} \geq \cdots \geq \lambda_{m} \geq 0$ ) we denote by $W_{m}(\lambda)$ the irreducible $\mathrm{GL}_{m}$-module corresponding to $\lambda$. The vector space $L_{m}$ is multigraded in a natural way counting the entries of each variable $x_{i}$ in the commutators from $L_{m}$. Every $\mathrm{GL}_{m}$-submodule of $L_{m}$ is a graded subspace, and its Hilbert (or Poincaré) series is

$$
H\left(W, t_{1}, \ldots, t_{m}, t\right)=\sum W^{\mathrm{n}} t_{1}^{n_{1}} \cdots t_{m}^{n_{m}} t^{n},
$$

where $\mathbf{n}=\left(n_{1}, \ldots, n_{m}\right), n_{1}+\cdots+n_{m}=n$, and $W^{\mathbf{n}}$ is the multihomogeneous component of $W$ of degree $n_{i}$ in $x_{i}$. The Hilbert series of $W$ is the character of the $\mathrm{GL}_{m}$-module. If $g \in \mathrm{GL}_{m}$ has eigenvalues $\xi_{1}, \ldots, \xi_{m}$, then

$$
\chi_{W}(g, t)=H\left(W, \xi_{1}, \ldots, \xi_{m}, t\right)=\sum \operatorname{tr}_{W^{(n)}}(g) t^{n},
$$

where $\operatorname{tr}_{W^{(n)}}(g)$ is the trace of $g$ acting on the homogeneous component of degree $n$ of $W$. Let $G$ be a finite subgroup of $\mathrm{GL}_{m}$. There is an analogue of the classical Molien formula for the invariants of a finite group on a $\mathrm{GL}_{m^{-}}$ submodule of $K\left\langle x_{1}, \ldots, x_{m}\right\rangle$ and hence on $L_{m}$.

Proposition 1.1 [5, Theorem 7]. Let char $K=0$, let $W$ be a $\mathrm{GL}_{m}$-submodule of $L_{m}$ with Hilbert series $H\left(W, t_{1}, \ldots, t_{m}, t\right)$, and let $G$ be a finite subgroup 
of $\mathrm{GL}_{m}$. The graded vector space $W^{G}=\{w \in W \mid g(w)=w$ for all $g \in G\}$ has a Hilbert series

$$
H\left(W^{G}, t\right)=\frac{1}{|G|} \sum_{g \in G} \chi_{W}(g, t) .
$$

Proposition 1.2. Let $\operatorname{char} K=p \neq 0$, and let the variety $\mathfrak{U}$ of Lie algebras over $K$ be defined by multilinear polynomial identities.

(i) The relatively free algebra $F_{m}(\mathfrak{U})$ is a multigraded vector space, and its Hilbert series $H\left(F_{m}(\mathfrak{U}), t_{1}, \ldots, t_{m}, t\right)$ is the $K$-character of the $\mathrm{GL}_{m}$-module $F_{m}(\mathfrak{U})$. If $g \in \mathrm{GL}_{m}$ has eigenvalues $\xi_{1}, \ldots, \xi_{m}$, then in $K[[t]]$

$$
\chi_{F_{m}(\mathfrak{U})}(g, t)=\operatorname{tr}_{F_{m}(\mathfrak{U})}(g, t)=H\left(F_{m}(\mathfrak{U}), \xi_{1}, \ldots, \xi_{m}, t\right) .
$$

(ii) If $G$ is a finite subgroup of $\mathrm{GL}_{m}$ and $p$ does not divide $|G|$, then

$$
H\left(F_{m}(\mathfrak{U})^{G}, t\right) \equiv \frac{1}{|G|} \sum_{g \in G} \chi_{F_{m}(\mathfrak{U})}(g, t) \quad(\bmod p),
$$

where the congruence modulo $p$ means that the formal power series are equal as elements in $(\mathbb{Z} / p \mathbb{Z})[[t]]$.

Proof. Let $\mathfrak{U}$ be defined by the multilinear polynomial identities $f_{i}\left(x_{1}, \ldots, x_{n_{i}}\right)$ $=0, i=1,2, \ldots$. Fixing a basis $y_{1}, \ldots, y_{m}$ of $V_{m}$, the verbal ideal $U$ of $L_{m}$ corresponding to $\mathfrak{U}$ is spanned on

$$
\left\{f_{i}\left(u_{1}, \ldots, u_{n_{i}}\right) \text { ad } w_{1} \cdots \text { ad } w_{q_{i}} \mid u_{k}, w_{l} \text { commutators of } y_{1}, \ldots, y_{m}\right\} \text {. }
$$

Hence $F_{m}(\mathfrak{U})$ is a multigraded vector space with respect to any basis $\left\{y_{1}, \ldots\right.$, $\left.y_{m}\right\}$. If $\widetilde{K}$ is an extension of the base field $K$, the $\widetilde{K}$-algebra $\widetilde{L}_{m}=\widetilde{K} \otimes_{K}$ $L_{m}$ is the free Lie algebra generated by $\tilde{x}_{i}=1 \otimes x_{i}, i=1, \ldots, m$, and $\widetilde{F}_{m}(\tilde{\mathfrak{U}})=\widetilde{K} \otimes_{K} F_{m}(\mathfrak{U})$ is the relatively free algebra of the variety $\widetilde{\mathfrak{U}}$ of $\widetilde{K}$ algebras defined by the polynomial identities $\tilde{f}_{i}=1 \otimes f_{i}=0, i=1,2, \ldots$. Let $g \in \mathrm{GL}_{m}$. Considering an extension $\widetilde{K}$ of $K$ we may assume that $g$ acts as an upper triangular matrix on $\widetilde{V}_{m}=\widetilde{K} \otimes_{K} V_{m}$. The Hilbert series of $F_{m}(\mathfrak{U})$ is independent of the choice of the basis of $V_{m}$. Hence

$$
\operatorname{tr}_{F_{m}(\mathfrak{U})}(g, t)=H\left(F_{m}(\mathfrak{U}), \xi_{1}, \ldots, \xi_{m}, t\right) .
$$

(ii) For any finite-dimensional $G$-module $W$ the linear operator

$$
\pi: w \rightarrow \frac{1}{|G|} \sum_{g \in G} g(w), \quad w \in W,
$$

projects $W$ onto $W^{G}$. Hence $\operatorname{tr}_{W}(\pi) \equiv \operatorname{dim} W^{G}(\bmod p)$, and this completes the proof because $F_{m}(\mathfrak{U})^{G}$ is a direct sum of its homogeneous components of degree $n=1,2, \ldots$.

We denote by $\mathfrak{A}^{2}$ the metabelian variety of Lie algebras defined by the polynomial identity $\left[\left[x_{1}, x_{2}\right],\left[x_{3}, x_{4}\right]\right]=0$. Clearly $F_{m}\left(\mathfrak{A}^{2}\right)=L_{m} / L_{m}^{\prime \prime}$.

Proposition 1.3. Over an arbitrary field,

$$
\begin{aligned}
& H\left(F_{m}\left(\mathfrak{A}^{2}\right), t_{1}, \ldots, t_{m}, t\right) \\
& \quad=1+\left(t_{1}+\cdots+t_{m}\right) t+\left(\left(t_{1}+\cdots+t_{m}\right) t-1\right) \prod_{i=1}^{m} \frac{1}{1-t_{i} t} .
\end{aligned}
$$


Proof. The algebra $F_{m}\left(\mathfrak{A}^{2}\right)$ has a basis

$$
x_{i},\left[x_{i_{1}}, x_{i_{2}}, \ldots, x_{i_{n}}\right], \quad i_{1}>i_{2} \leq \cdots \leq i_{n}, n=2,3, \ldots
$$

(see, e.g., [8, §2, pp. 274-276 of the English translation] where bases of the free polynilpotent Lie algebras are found). Since the basis of $F_{m}\left(\mathfrak{A}^{2}\right)$ does not depend on the characteristic of the field, we may assume that char $K=0$. It is well known (see, e.g., [3, Proof of Lemma 2.5]) that as a $\mathrm{GL}_{m}$-module

$$
F_{m}\left(\mathfrak{A}^{2}\right) \cong W_{m}(1)+\sum_{n \geq 2} W_{m}(n-1,1) .
$$

The character of the irreducible $\mathrm{GL}_{m}$-module $W_{m}(\lambda)$ is equal to the Schur function $S_{\lambda}\left(t_{1}, \ldots, t_{m}\right)$. For $\lambda=(n)$ the Schur function $S_{(n)}\left(t_{1}, \ldots, t_{m}\right)$ is equal to the $n$th complete symmetric function $h_{n}\left(t_{1}, \ldots, t_{m}\right)$. By [7, Chapter $1,5.15$, p. 42], for $n \geq 1$

$$
\begin{aligned}
h_{n}\left(t_{1}, \ldots, t_{m}\right) h_{1}\left(t_{1}, \ldots, t_{m}\right) & =S_{(n)}\left(t_{1}, \ldots, t_{m}\right) S_{(1)}\left(t_{1}, \ldots, t_{m}\right) \\
& =S_{(n+1)}\left(t_{1}, \ldots, t_{m}\right)+S_{(n, 1)}\left(t_{1}, \ldots, t_{m}\right) \\
& =h_{n+1}\left(t_{1}, \ldots, t_{m}\right)+S_{(n, 1)}\left(t_{1}, \ldots, t_{m}\right),
\end{aligned}
$$

$$
\begin{aligned}
H & \left.F_{m}\left(\mathfrak{A}^{2}\right), t_{1}, \ldots, t_{m}, t\right) \\
& =S_{(1)}\left(t_{1}, \ldots, t_{m}\right) t+\sum_{n \geq 2} S_{(n-1,1)}\left(t_{1}, \ldots, t_{m}\right) t^{n} \\
& =h_{1}\left(t_{1}, \ldots, t_{m}\right) t+\sum_{n \geq 2}\left(h_{n-1}\left(t_{1}, \ldots, t_{m}\right) h_{1}\left(t_{1}, \ldots, t_{m}\right)-h_{n}\left(t_{1}, \ldots, t_{m}\right)\right) t^{n} \\
& =h_{1}\left(t_{1}, \ldots, t_{m}\right) t\left(1+\sum_{n \geq 1} h_{n}\left(t_{1}, \ldots, t_{m}\right) t^{n}\right)-\sum_{n \geq 2} h_{n}\left(t_{1}, \ldots, t_{m}\right) t^{n} \\
& =\left(h_{1}\left(t_{1}, \ldots, t_{m}\right) t-1\right) \sum_{n \geq 0} h_{n}\left(t_{1}, \ldots, t_{m}\right) t^{n}+1+h_{1}\left(t_{1}, \ldots, t_{m}\right) t,
\end{aligned}
$$

and the assertion follows because

$$
h_{1}\left(t_{1}, \ldots, t_{m}\right)=t_{1}+\cdots+t_{m}, \quad \sum_{n \geq 0} h_{n}\left(t_{1}, \ldots, t_{m}\right) t^{n}=\prod_{i=1}^{m} \frac{1}{1-t_{i} t} .
$$

Proposition 1.4. If $G$ is a finite subgroup of Aut $F_{m}\left(\mathfrak{A}^{2}\right), m>1$, and the order of $G$ is invertible in $K$, then the fixed algebra $F_{m}\left(\mathfrak{A}^{2}\right)^{G}$ is infinite dimensional. Proof. First, let $G$ be a subgroup of $\mathrm{GL}_{m}$. If $F_{m}\left(\mathfrak{A}^{2}\right)^{G}$ is finite dimensional, then its Hilbert series $H\left(F_{m}\left(\mathfrak{A}^{2}\right)^{G}, t\right)$ is a polynomial $f(t)$. By Propositions 1.1 and 1.2

$$
H\left(F_{m}\left(\mathfrak{A}^{2}\right)^{G}, t\right)=\frac{1}{|G|} \sum_{g \in G} \chi_{F_{m}\left(\mathfrak{A}^{2}\right)}(g, t),
$$

where the equality is replaced by a congruence modulo $p$ if $\operatorname{char} K=p \neq 0$. Let $g \in G$ have characteristic roots $\xi_{1}, \ldots, \xi_{m}$. Then

$$
\xi_{1}+\cdots+\xi_{m}=\operatorname{tr} g, \quad \prod_{i=1}^{m}\left(1-\xi_{i} t\right)=\operatorname{det}(1-t g),
$$




$$
\begin{gathered}
H\left(F_{m}\left(\mathfrak{A}^{2}\right)^{G}, t\right)=1+\frac{1}{|G|} \sum_{g \in G}\left((\operatorname{tr} g) t+\frac{(\operatorname{tr} g) t-1}{\operatorname{det}(1-\operatorname{tg})}\right)=f(t), \\
\sum_{g \in G} \frac{(\operatorname{tr} g) t-1}{\operatorname{det}(1-t g)}=|G|(f(t)-1)-\sum_{g \in G}(\operatorname{tr} g) t=f_{1}(t),
\end{gathered}
$$

where $f_{1}(t)$ is a polynomial and $\operatorname{det}^{-1}(1-t g)$ is a short notation for the formal series $\prod_{i=1}^{m}\left(\sum_{n \geq 0} \xi_{i}^{n} t^{n}\right)$ if char $K \neq 0$. Replacing $t$ by 0 we obtain that $f_{1}(0)=-|G| \neq 0$. Obviously $\operatorname{det}(1-t g)$ is a polynomial of degree $m$. We compare the degrees of both the sides of the equality

$$
\left(\prod_{g \in G} \operatorname{det}(1-t g)\right) \sum_{g \in G} \frac{(\operatorname{tr} g) t-1}{\operatorname{det}(1-t g)}=\left(\prod_{g \in G} \operatorname{det}(1-t g)\right) f_{1}(t)
$$

and obtain that the right-hand side is of degree $\geq|G| m$ while the left-hand side is of degree $\leq 1+(|G|-1) m$. Since $m>1$, this is impossible; i.e., $H\left(F_{m}\left(\mathfrak{A}^{2}\right)^{G}, t\right)$ is not a polynomial. In the general case, let $G \subset$ Aut $F_{m}\left(\mathfrak{A}^{2}\right)$ and let $\operatorname{dim} F_{m}\left(\mathfrak{A}^{2}\right)<\infty$. Hence the elements of $F_{m}\left(\mathfrak{A}^{2}\right)^{G}$ are of degree bounded by an integer $n_{0}$. The map $\rho: G \rightarrow \mathrm{GL}_{m}$ defined by

$$
(\rho(g))(v) \equiv g(v)\left(\bmod F_{m}^{\prime}\left(\mathfrak{A}^{2}\right)\right), \quad v \in V_{m}, \quad g \in G,
$$

is a group homomorphism. We consider the algebra of invariants $F_{m}\left(\mathfrak{A}^{2}\right)^{\rho(G)}$ of the linear group $\rho(G)$ acting on $F_{m}\left(\mathfrak{A}^{2}\right)$. Since $F_{m}\left(\mathfrak{A}^{2}\right)^{\rho(G)}$ is not finite dimensional, there exists a homogeneous $f\left(x_{1}, \ldots, x_{m}\right) \in F_{m}\left(\mathfrak{A}^{2}\right)^{\rho(G)}$ of degree $n$ greater than $n_{0}$. Obviously

$(\rho(g))\left(f\left(x_{1}, \ldots, x_{m}\right)\right) \equiv g\left(f\left(x_{1}, \ldots, x_{m}\right)\right) \quad\left(\bmod F_{m}^{n+1}\left(\mathfrak{A}^{2}\right)\right)$,
$h=\frac{1}{|G|} \sum_{g \in G} g(f) \in F_{m}\left(\mathfrak{A}^{2}\right)^{G}, \quad h \equiv \frac{1}{|G|} \sum_{g \in G}(\rho(g))(f)=f \quad\left(\bmod F_{m}^{n+1}\left(\mathfrak{A}^{2}\right)\right)$,

$h \neq 0$, and $\operatorname{deg} h \geq n$. Hence $\operatorname{dim} F_{m}\left(\mathfrak{A}^{2}\right)^{G}=\infty$.

\section{The MAIN RESUlTS}

Lemma 2.1. Let $R$ be a residually nilpotent Lie algebra generated by $r_{1}, \ldots, r_{m}$, and let $r_{1}, \ldots, r_{m}$ be linearly independent modulo $R^{\prime}$. If $G$ is a finite subgroup of Aut $R$ of order invertible in $K$, then the canonical homomorphism $\rho: G \rightarrow \mathrm{GL}\left(R / R^{\prime}\right)$ is injective.

Proof. Every automorphism $\phi$ of $R$ is determined by $\phi\left(r_{i}\right), i=1, \ldots, m$. The ideal $R^{n}$ is closed under all endomorphisms of $R$; the map $\rho_{n}: G \rightarrow$ $\operatorname{Aut}\left(R / R^{n+1}\right)$ defined by

$$
\left(\rho_{n}(g)\right)\left(r_{i}\right) \equiv g\left(r_{i}\right)\left(\bmod R^{n+1}\right), \quad i=1, \ldots, m,
$$

is a group homomorphism; $\rho=\rho_{1}$; and $G \supset \operatorname{Ker} \rho_{1} \supset \operatorname{Ker} \rho_{2} \supset \cdots$. The algebra $R$ is residually nilpotent, i.e., $\bigcap_{n \geq 1} R^{n}=\{0\}$, and $\bigcap_{n \geq 1} \operatorname{Ker} \rho_{n}=\langle 1\rangle$. If $g \in \operatorname{Ker} \rho_{n} \backslash \operatorname{Ker} \rho_{n+1}, n \geq 1$, then $g\left(r_{i}\right)=r_{i}+f_{i}$, where $f_{i}=f_{i}\left(r_{1}, \ldots, r_{m}\right) \in$ $R^{n+1}, i=1, \ldots, m$, and $f_{i_{0}}$ is not contained in $R^{n+2}$ for some $i_{0}$. It is easy to see that

$$
g^{k}\left(r_{i_{0}}\right) \equiv r_{i_{0}}+k f_{i_{0}}\left(r_{1}, \ldots, r_{m}\right) \quad\left(\bmod R^{n+2}\right) .
$$


If char $K=0$, then $g^{k} \neq 1$ for all $k \geq 1$, which is impossible because $|G|<\infty$. If char $K=p \neq 0$, then $g^{k}\left(r_{i_{0}}\right) \equiv r_{i_{0}}\left(\bmod R^{n+2}\right)$ if and only if $p$ divides $k$. Hence $|G|$ is not invertible in $K$, which is a contradiction, and $\operatorname{Ker} \rho_{n}=\langle 1\rangle$ for all $n \geq 1$.

Lemma 2.2. Every finite subset of $F_{m}\left(\mathfrak{A}^{2}\right)$ which is linearly independent modulo $F_{m}^{\prime}\left(\mathfrak{A}^{2}\right)$ generates a free metabelian subalgebra of $F_{m}\left(\mathfrak{A}^{2}\right)$.

Proof. The following arguments hold for any variety $\mathfrak{U}$ defined by homogeneous polynomial identities. Let $w_{1}, \ldots, w_{q} \in F_{m}\left(\mathfrak{A}^{2}\right)$ be linearly independent modulo $F_{m}^{\prime}\left(\mathfrak{A}^{2}\right)$. Applying an invertible linear transformation to the free generators of $F_{m}\left(\mathfrak{A}^{2}\right)$ we may assume that $w_{i} \equiv x_{i}\left(\bmod F_{m}^{\prime}\left(\mathfrak{A}^{2}\right)\right), i=1, \ldots, q$. Let $0 \neq f\left(x_{1}, \ldots, x_{r}\right) \in F_{m}^{k}\left(\mathfrak{A}^{2}\right) \backslash F_{m}^{k+1}\left(\mathfrak{A}^{2}\right)$. Since

$$
f\left(w_{1}, \ldots, w_{q}\right) \equiv f\left(x_{1}, \ldots, x_{q}\right) \not \equiv 0 \quad\left(\bmod F_{m}^{k+1}\left(\mathfrak{A}^{2}\right)\right),
$$

we obtain that all the relations between $w_{1}, \ldots, w_{q}$ follow from the metabelian identity, i.e., $w_{1}, \ldots, w_{q}$ generate a free metabelian algebra.

Theorem 2.3. Let $R=F_{m}\left(\mathfrak{A}^{2}\right)=L_{m} / L_{m}^{\prime \prime}$ be the free metabelian Lie algebra of rank $m>1$. If $G \neq\langle 1\rangle$ is a finite subgroup of Aut $R$ of order invertible in the base field $K$, then the fixed algebra $F_{m}\left(\mathfrak{A}^{2}\right)^{G}$ is not finitely generated.

Proof. The ideal $F_{m}^{\prime}\left(\mathfrak{A}^{2}\right)$ is a $G$-module and by the Maschke theorem there exist $G$-submodules $W$ and $Z$ of $F_{m}\left(\mathfrak{A}^{2}\right)$ such that

$$
F_{m}\left(\mathfrak{A}^{2}\right)=W \oplus Z \oplus F_{m}^{\prime}\left(\mathfrak{A}^{2}\right), \quad(W \oplus Z)^{G}=Z, \quad \operatorname{dim}(W \oplus Z)=m .
$$

We fix bases $\left\{w_{1}, \ldots, w_{q}\right\}$ of $W$ and $\left\{z_{q+1}, \ldots, z_{m}\right\}$ of $Z$. We may assume that

$$
w_{i} \equiv x_{i}, \quad z_{j} \equiv x_{j}\left(\bmod F_{m}^{\prime}\left(\mathfrak{A}^{2}\right)\right), \quad i=1, \ldots, q, j=q+1, \ldots, m .
$$

If $q=0$, then $G$ acts on $F_{m}\left(\mathfrak{A}^{2}\right)$ trivially modulo $F_{m}^{\prime}\left(\mathfrak{A}^{2}\right)$ and, by Lemma 2.1, $G=\langle 1\rangle$. Hence $q>0$. Let the algebra $F_{m}\left(\mathfrak{A}^{2}\right)^{G}$ be finitely generated. As a vector space $F_{m}\left(\mathfrak{A}^{2}\right)^{G}$ is a direct sum of $Z$ and $F_{m}^{\prime}\left(\mathfrak{A}^{2}\right)^{G}$, and hence $F_{m}\left(\mathfrak{A}^{2}\right)^{G}$ is generated as an algebra by $z_{q+1}, \ldots, z_{m}$ and some $f_{1}, \ldots, f_{r} \in F_{m}^{\prime}\left(\mathfrak{A}^{2}\right)^{G}$. Since the $f_{i}$ 's are in $F_{m}^{\prime}\left(\mathfrak{A}^{2}\right)$, every element of $F_{m}^{\prime}\left(\mathfrak{A}^{2}\right)^{G}$ is of the form

$$
f=\sum\left(\alpha_{i k} f_{i}+\beta_{j k}\left[z_{j_{1}}, z_{j_{2}}\right]\right) \mathrm{ad}^{k_{q+1}} z_{q+1} \cdots \mathrm{ad}^{k_{m}} z_{m} .
$$

The operators ad $z_{j}$ and ad $x_{j}$ are equal on $F_{m}^{\prime}\left(\mathfrak{A}^{2}\right)$ and

$$
f=\sum\left(\alpha_{i k} f_{i}+\beta_{j k}\left[z_{j_{1}}, z_{j_{2}}\right]\right) \mathrm{ad}^{k_{q+1}} x_{q+1} \cdots \mathrm{ad}^{k_{m}} x_{m},
$$

i.e., the elements of $F_{m}\left(\mathfrak{A}^{2}\right)^{G}$ are of bounded degree in $x_{1}, \ldots, x_{q}$.

Case 1. $q>1$. The group $G$ acts on the algebra $\langle W\rangle$ generated by $w_{1}, \ldots, w_{q}$ as a group of automorphisms, and the vector space $W$ has no fixed points. By Lemma 2.2, $\langle W\rangle \cong F_{q}\left(\mathfrak{A}^{2}\right)$, and by Proposition $1.4\langle W\rangle^{G}$ is infinite dimensional. Since for $f \in\langle W\rangle^{G}$

$$
\begin{aligned}
f & =\sum \alpha_{i k}\left[w_{i_{1}}, w_{i_{2}}\right] \operatorname{ad}^{k_{1}} w_{1} \cdots \operatorname{ad}^{k_{q}} w_{q} \\
& =\sum \alpha_{i k}\left[w_{i_{1}}, w_{i_{2}}\right] \operatorname{ad}^{k_{1}} x_{1} \cdots \operatorname{ad}^{k_{q}} x_{q} \in F_{m}\left(\mathfrak{A}^{2}\right)^{G},
\end{aligned}
$$


the degrees in $x_{1}, \ldots, x_{q}$ of the elements from $F_{m}\left(\mathfrak{A}^{2}\right)^{G}$ are not bounded, which is a contradiction.

Case 2. $q=1$. Every element $g \in G$ acts on $w_{1}$ by scalar multiplication, i.e., $g\left(w_{1}\right)=\xi_{g} w_{1}$ and $\xi_{g}^{n}=1, n=|G|$. Hence

$$
g\left(z_{m} \operatorname{ad}^{n k} w_{1}\right)=\xi_{g}^{n k} z_{m} \operatorname{ad}^{n k} w_{1}=z_{m} \operatorname{ad}^{n k} w_{1}, \quad g \in G, k=1,2, \ldots,
$$

and $z_{m} \operatorname{ad}^{n k} w_{1} \in F_{m}\left(\mathfrak{A}^{2}\right)^{G}$. Again $z_{m} \operatorname{ad}^{n k} w_{1}=\left[z_{m}, w_{1}\right] \operatorname{ad}^{n k-1} x_{1}$ and there exists no upper bound for the degree in $x_{1}$ of the elements from $F_{m}\left(\mathfrak{A}^{2}\right)^{G}$.

Theorem 2.4. Let $L_{m}$ be the free Lie algebra of rank $m>1$ over a field $K$, and let $J$ be an ideal of $L_{m}$ such that $J \subset L_{m}^{\prime \prime}$ and the algebra $L_{m} / J$ is residually nilpotent. If $G \neq\langle 1\rangle$ is a finite group of automorphisms of $L_{m} / J$ of order invertible in $K$, then the algebra of fixed points $\left(L_{m} / J\right)^{G}$ is not finitely generated.

Proof. Every automorphism of $R=L_{m} / J$ induces an automorphism on $R / R^{\prime \prime}$. By Lemma 2.1 the group $G$ acts faithfully on $R / R^{\prime}$ and hence on $R / R^{\prime \prime}$. Now $J \subset L_{m}^{\prime \prime}$ implies that $R / R^{\prime \prime} \cong L_{m} / L_{m}^{\prime \prime} \cong F_{m}\left(\mathfrak{A}^{2}\right)$. The map $R^{G} \rightarrow\left(R / R^{\prime \prime}\right)^{G}$ is surjective because $|G|$ is invertible in $K$. If $R^{G}$ is finitely generated, the same holds for $\left(R / R^{\prime \prime}\right)^{G}$, and this is in contradiction with Theorem 2.3.

Corollary 2.5. If $G \neq\langle 1\rangle$ is a finite subgroup of Aut $L_{m}, m>1$, and $|G|$ is invertible in $K$, then $L_{m}^{G}$ is not finitely generated.

Theorem 2.6. Let the field $K$ be infinite, char $K \neq 2,3$, and let $\mathfrak{U}$ be a variety of Lie algebras over $K$. For a finite subgroup $G \neq\langle 1\rangle$ of Aut $F_{m}(\mathfrak{U}), m>1$, the fixed algebra $F_{m}(\mathfrak{U})^{G}$ is finitely generated if and only if $\mathfrak{U}$ is nilpotent.

Proof. Let $U \subset L_{m}$ be the verbal ideal corresponding to $\mathfrak{U}$. Since the base field is infinite, the algebra $F_{m}(\mathfrak{U})$ is multihomogeneous and hence residually nilpotent. It is well known that either $\mathfrak{U}$ satisfies the Engel identity $x_{2}\left(\operatorname{ad}^{n} x_{1}\right)=$ 0 for some $n>0$ or $U \subset L_{m}^{\prime \prime}$. If the variety $\mathfrak{U}$ satisfies $x_{2}\left(\operatorname{ad}^{n} x_{1}\right)=0$, by the theorem of Zel'manov [9], $\mathfrak{U}$ is nilpotent. Hence $F_{m}(\mathfrak{U})$ is finite dimensional and $F_{m}(\mathfrak{U})^{G}$ is finitely generated. If $U \subset L_{m}^{\prime \prime}$ we apply Theorem 2.3.

\section{ACKNOWLEDGMENTS}

The author is very grateful to C. K. Gupta who paid attention to the problem for finite generation of the fixed algebra of the free Lie algebra which was the starting point of this research.

\section{REFERENCES}

1. A. I. Belov, Periodic automorphisms of free Lie algebras and their fixed points, preprint.

2. W. Dicks and E. Formanek, Poincaré series and a problem of S. Montgomery, Linear and Multilinear Algebra 12 (1982), 21-30.

3. V. Drensky and A. Kasparian, Polynomial identities of eighth degree for $3 \times 3$ matrices, Ann. Univ. Sofia Fac. Math. Méc. 77 (1983), 175-195.

4. J. L. Dyer and G. P. Scott, Periodic automorphisms of free groups, Comm. Algebra 3 (1975), 195-201.

5. E. Formanek, Noncommutative invariant theory, Group Actions on Rings, Contemp. Math., vol. 43, Amer. Math. Soc., Providence, RI, 1985, pp. 87-119. 
6. V. K. Kharchenko, Noncommutative invariants of finite groups and Noetherian varieties, J. Pure Appl. Algebra 31 (1984), 83-90.

7. I. G. Macdonald, Symmetric functions and Hall polynomials, Oxford Univ. Press, Clarendon, Oxford, 1979.

8. A. L. Shmel'kin, Free polynilpotent groups, Izv. Akad. Nauk SSSR Ser. Mat. 28 (1964), 91-122; English transl. Amer. Math. Soc. Transl. Ser. 2, vol. 55, Amer. Math. Soc., Providence, RI, 1966, pp. 270-304.

9. E. I. Zelmanov, Weakened Burnside problem, Sibirsk. Math. Zh. 30 (1989), 68-74; English transl. Siberian Math. J. 30 (1989), 885-891.

Institute of Mathematics, Bulgarian Academy of Sciences, Acad. G. Bonchev Str., BLock 8, 1113 Sofia, Bulgaria 